\title{
Postinfection irritable bowel syndrome
}

\section{Marco Marcello Marcellini, Maria Raffaella Barbaro, Cesare Cremon and Giovanni Barbara*}

Department of Medical and Surgical Sciences, University of Bologna, St. Orsola-Malpighi Hospital, Bologna, Italy

\section{RESUMEN}

El síndrome del intestino irritable (IBS) es un trastorno gastrointestinal multifactorial. Un episodio agudo de enteritis infecciosa es uno de los factores de riesgo más relevantes para el desarrollo de IBS, el denominado IBS post-infección IBS (PIIBS). En este artículo pretendemos revisar las publicaciones relevantes sobre la relación entre la gastroenteritis infecciosa y el consiguiente desarrollo de IBS, los factores de riesgo relacionados para el desarrollo de PIIBS, los principales patógenos involucrados, el papel de la microbiota y las terapias. Hemos tomado en consideración los últimos estudios indexados en Pubmed utilizando los siguientes ítems de búsqueda: «IBS», «PIIBS», «therapy of PIIBS». Los resultados de nuestra revisión mostraron que la incidencia de PIIBS oscila entre el 3 y el 30\% después de la gastroenteritis infecciosa. Aunque la gastroenteritis por protozoos y parásitos se asoció con el desarrollo de PIIBS en hasta un 41.9\% de los casos en comparación con solo un $13.8 \%$ después de infecciones bacterianas; se necesitan más estudios para confirmar estas cifras. No hay un tratamiento específico de PIIBS y los enfoques principales reflejan los utilizados para el IBS. En conclusión, la gastroenteritis por infección aguda es un factor de riesgo para el desarrollo de IBS de nueva aparición. Los estudios futuros deberían centrarse en los mecanismos implicados en esta conexión con el fin de desarrollar estrategias terapéuticas que prevengan la aparición de nuevos IBS.

Palabras clave: Síndrome del intestino irritable. Infección. Microbiota. Probióticos. 


\section{ABSTRACT}

Irritable bowel syndrome (IBS) is a multifactorial gastrointestinal disorder. An acute episode of infectious enteritis is one of the most relevant risk factors for the development of IBS, the so-called postinfection IBS (PIIBS). Here, we aim to review the relevant publications on the relationship between infectious gastroenteritis and the consequent development of IBS, the related risk factors for PIIBS development, the main pathogens involved, the role of microbiota, and therapies. We have taken into consideration the latest studies indexed on PubMed using the following searching items: "IBS," "PIIBS," and "Therapy of PIIBS." The results of our review showed that the incidence of PIIBS ranges between $3 \%$ and $30 \%$ after infectious gastroenteritis. Although protozoa and parasites gastroenteritis were associated with the development of PIIBS in as high as $41.9 \%$ of cases compared to only $13.8 \%$ following bacterial infections, further studies are needed to confirm these figures. There is no specific treatment of PIIBS, and main approaches mirror those utilized for IBS. In conclusion, acute infection gastroenteritis is a risk factor for the development of new-onset IBS. Future studies should focus on the mechanisms involved in this connection to develop therapeutic strategies preventing the onset of new IBS. (NeuroGastroLatam Rev. 2018;2:78-86).

Corresponding author: Giovanni Barbara, giovanni.barbara@unibo.it

Key words: Irritable bowel syndrome. Infection. Microbiota. Probiotics.

\section{INTRODUCTION}

The irritable bowel syndrome (IBS) is one of the most common gastrointestinal disorders characterized by recurrent abdominal pain, on average, at least 1 day per week in the past 3 months, associated with two or more of the following criteria: (a) related to defecation; (b) associated with a change in frequency of stool; and (c) associated with a change in the form (appearance) of stool. Criteria need to be fulfilled for the past 3 months with symptom onset at least 6 months before diagnosis ${ }^{1}$. Although these symptoms occur in the absence of organic or biochemical abnormalities, several recent studies have provided evidence of microscopic or molecular changes. These include immune cell infiltration (i.e., $\mathrm{T}$ cells and mast cells) and immune activation (e.g., release of cytokines, histamine, and proteases) in the intestinal mucosa of IBS patients. Recent findings suggest that mucosal immune activation could participate in altered bowel physiology, increased visceral sensitivity, and symptom perception ${ }^{2}$.

Established evidence indicates that acute infectious gastroenteritis of diverse etiology including bacteria, viruses, protozoa, and parasites $^{3}$ may lead, in about one in $10 \mathrm{sub-}$ jects, to the development of persistent gastrointestinal symptoms, once the infection has subsided and the infective agent has been eliminated from the gut. In many of these subjects, symptoms may fulfill the criteria for IBS, a condition known as 
postinfection (PI) IBS ${ }^{4,5}$. Patients with PIIBS have been reported to have increased mucosal production of pro-inflammatory cytokines such as interleukin (IL)-1 and IL-6, reflecting a state of persistent low-grade inflammation $^{6}$. The gut microbiota, a major driver of host's mucosal immune function ${ }^{2}$, may be dysregulated with reduced biodiversity ${ }^{7}$ and an increase in bacterial groups associated with mucosal inflammation ${ }^{8}$. The changes in gut microbiota described in PIIBS are similar to those seen in patients with IBS with diarrhea $\left(\right.$ IBS-D) ${ }^{8}$. Gut dysbiosis has been suggested to be a pathogenetic factor involved in the low-grade immune activation seen in PIIBS. Indeed, there is a direct correlation between reduced microbial diversity and the degree of mucosal immune activation seen in PIIBS7. In addition, changes in gut microbial communities in PIIBS are associated with abnormal expression of host tissue genes involved in the regulation of mucosal permeability and immune activation ${ }^{8}$. In this context, increased intestinal permeability would expose the mucosal immune system to an increased antigenic load with stimulation of exaggerated immune responses. In addition, the inflammatory response would maintain increased intestinal permeability creating a vicious circle ${ }^{8}$.

In this review, we will report on the current evidence supporting a relationship between acute infection gastroenteritis and the new development of IBS symptoms. We will deal with the main aspects of PIIBS on epidemiology, risk factors, pathophysiology, including the role of microbiota, immune function, and intestinal permeability as well as aspects of the management of these patients.

\section{EPIDEMIOLOGY}

In a recent meta-analysis of 45 studies including 21,421 patients, the pooled prevalence of IBS after an acute episode of infectious enteritis was $10.1 \%$ at 12 months and $14.5 \%$ at more than 12 months $^{3}$. There are limited data assessing differences in the prevalence of PIIBS between the northern and the southern hemisphere.

The estimated risk of occurrence of IBS in patients who had an acute infectious enteritis was 4.2-fold higher compared to healthy controls. The etiology of the infection has been reported to have a strong influence on the development of IBS. Indeed, protozoa or parasites were associated with the development of IBS in up to $41.9 \%$ of cases compared to bacterial infections associated only to $13.8 \%$. Other commonly associated risk factors were female sex, antibiotic treatment, and psychological factors such as anxiety, depression, and somatization ${ }^{3}$.

Age of subjects at the time of acute infection is another risk factor reported in many studies $^{9-11}$. Saps et al. studied a sample of 88 children with infectious gastroenteritis caused by Campylobacter, Salmonella, and Shigella. Children were prone to develop both PIIBS and PI functional dyspepsia as chronic symptoms could be recorded in as many as $56 \%$ of these subjects ${ }^{9}$. In a large outbreak of Salmonella gastroenteritis, Cremon et al. showed that 16 years after the acute infection, $35.3 \%$ of exposed compared to $20.5 \%$ of non-exposed subjects developed IBS. In this study, it was confirmed that children were at higher risk compared to adults to develop PIIBS ${ }^{10}$. The 
reasons for a higher prevalence of PIIBS in children compared to adults are far from being clarified. In infants and toddlers, the gut microbiota and the immune system are less developed and more susceptible to the effects of acute infections. Host defense mechanisms could be inefficient to control the acute infectious episode, leading to a more severe and penetrating infection. Indeed, the severity of infection gastroenteritis has been reported to be a risk factor for the development of PIIBS in several studies.

\section{PATHOGENS}

The living pathogenetic microorganisms that most often cause an infection of the gastrointestinal tract belong to the three main domains: eukaryotes (protozoa and parasites), prokaryotes (bacteria) ${ }^{12}$, and viruses ${ }^{13}$.

In a subgroup analysis of 12 controlled studies assessing the relative risk for PIIBS, the highest risk was observed with protozoal infections (RR 3.25; 95\% CI, 2.86-3-69). Eukaryote (protozoa and parasites) have been reported to be involved in several epidemics and to induce PIIBS in a high rate ${ }^{3}$. Eucarya are unicellular eukaryotic microorganisms, aerobic, and facultative anaerobes equipped with means of locomotion represented by pseudopodia or cilia or flagella, and they can survive and feed on the surface of mucous epithelia such as intestinal (Giardia lamblia) ${ }^{14}$. The lowest risk of developing PIIBS was associated with viral infections (RR 1.19; 95\% CI, 0.502.84), while bacterial etiologies, including, Escherichia coli, Salmonella enteritidis, and Campylobacter jejuni, were associated with an intermediate risk (RR 2.24; 95\% CI, 1.63-3.10) ${ }^{3}$.
The relative high risk of PIIBS after bacterial infections is related to bacterial pathogenicity linked to their invasiveness capacity, production of toxins, and damage of epithelial tight junctions, leading to neutrophil infiltration and a strong inflammatory reaction ${ }^{15,16}$. In contrast, the low incidence of PIIBS after viral infections could be explained by the fact that viral infections are relatively milder, of short duration and self limiting ${ }^{13,17}$. The high risk of developing PIIBS after protozoalinfections remains unclear.

\section{MICROBIOTA}

The human gut microbiota is an ecosystem consisting of trillions of bacteria, fungi and viruses, and plurality of strains. The main bacterial phyla making up to $90 \%$ of the total microbiota are Bacteroides and Firmicutes. One of the best-studied aspects of microbiota metabolism is the production of short-chain fatty acids (SCFAs), which are the major source of energy for colonocytes. Other fundamental functions of the microbiota include the synthesis of vitamins, the absorption of ions and electrolytes, the metabolism of primary bile acids, and the production of substances with antibiotic activity (hydrogen peroxide, bacteriocidins, and lactocidin). The mucosal barrier composed of mucus, epithelial cells with the tight junctions sealing the intercellular spaces between them, allows protection against the systemic spread of the microbiota. The microbiota also acts as an ally of our immune system in the development of systemic and local immunity.

Dysregulation of any of the above-mentioned aspects has been involved in the 
pathophysiology of $\mathrm{IBS}^{18}$. Among the causes that could determine this dysregulation, there are gastrointestinal infections, stressful emotional factors, use of antibiotics, and hyperactivation of the immune system, including allergies. Each of these alterations is, therefore, at the basis of the phenomenon of dysbiosis that is the imbalance between protective and harmful microbial species within the microbiota.

During an acute episode of gastrointestinal infection, the associated diarrhea produces a strong reduction in the total amount and diversity of the gut microbiota ${ }^{19}$, a reduced production of SCFA, and an increase in fecal $\mathrm{pH}$. These changes increase intestinal permeability, further contributing to mucosal inflammation $^{20}$. In addition, infections such as Giardia duodenalis and C. jejuni may have a direct effect on the distribution of gut microbiota, further contributing to dysbiosis ${ }^{21}$. A reduction of microbiota biodiversity hampers its defensive properties facilitating the recurrence of infectious episodes.

The fecal microbiota and its relationships with clinical features of patients with PIIBS have been evaluated in a study by Jalanka-Tuovinen et al. ${ }^{8}$ This study showed the presence of 27 bacterial groups that discriminated PIIBS microbiota from that of healthy controls, providing an index of microbial dysbiosis. The fecal microbiota of PIIBS was similar to that of IBS-D.

The fecal microbiota of PIIBS differed from that of healthy controls for the increase of various members of Bacteroides and decrease of uncultured Clostridiales. These microbial dysbiosis profiles were positively correlated with gastrointestinal but not psychological symptoms. In addition, these microbial profiles were correlated with host mucosal gene expression indicative of a dysregulation of intestinal barrier function and immune activation in PIIBS $^{8}$.

\section{RISK FACTORS FOR PIIBS}

\section{Female sex}

IBS is a disorder seen predominantly in females with a female:male ratio of 2:1. In addition, the majority of studies found that female gender is a risk factor (odds ratios ranging from 2 to 3) for the development of PIIBS. While there are several factors differentiating females from males, there is no current unifying theory on the increased risk of developing PIIBS in females. Among the most prevalent theories for the increased risk of IBS in females are hormonal factors, an increased prevalence of anxiety disorders and a greater visceral sensitivity in females compared with males ${ }^{22}$. In addition, there are likely differential structural properties of the intestine, between the two sexes. One study showed that female patients have a deregulation of cytoskeleton dynamics through TESK1/CFL1 pathways in the small bowel mucosa of IBS-D ${ }^{23}$. This new aspect could open new horizons of discussion aimed at reinforcing the concept that an altered intestinal permeability can be the background to IBS.

\section{ANTIBIOTIC EXPOSURE}

The use of systemic antibiotics during the acute infection has been suggested to 
increase the risk of developing PIIBS, but whether the increased risk is associated to a more severe illness or the presence of psychological factors is currently unknown. The potential mechanisms underlying the possible effect of antibiotics may include a negative effect of systemic antibiotics on gut microbiota ${ }^{24}$. Accordingly, systemic antibiotics may lead to overgrowth of pathobiont species residing in the gut such as fungi and Clostridium difficile, as well as in the diminished production of SCFAs essential in ion absorption and the survival of colonocytes $^{25,26}$. In contrast, poorly absorbable antibiotics such as rifaximin appear to improve IBS symptoms in non-constipated IBS patients, as demonstrated in randomized, placebo-controlled trials ${ }^{27,28}$ comprising more than 1200 patients. However, there are no specific controlled studies assessing the use of rifaximin in PIIBS. The mechanism underlying the beneficial effect of rifaximin in IBS has been ascribed to the reduction of pathobionts and the growth of beneficial bacterial groups in the gut, such as Lactobacilli and Bifidobacteria, as well as to anti-inflammatory properties ${ }^{29}$.

\section{ANXIETY, SOMATIZATION, AND DEPRESSION}

Functional gastrointestinal disorders including IBS have been recently redefined as disorders of gut-brain interaction, to reflect the involvement of both central and peripheral factors underlying symptom generation. Psychological distress is an important risk factor in symptom perception and the development of chronicity of IBS. In physiological conditions, in fact, intestinal peripheral stimuli, including painful stimuli, are constantly filtered by the brain in specific areas such as brainstem sensory nuclei, thalamus, and posterior insula. Subsequently they are further elaborated by the locus coeruleus, amygdala and prefrontal cortex. Nociceptive stimuli then return toward the periphery through the dorsal horn of the spinal cord where they are further modulated. During their passage through the central nervous system, the stimuli are crucially modulated by a psychological component. Subjects suffering from psychological stress manifest a poor management of the painful impulse, especially if linked to small stimuli ${ }^{30}$. Psychological distress has been suggested to play a key role in the pathogenesis of PIIBS. Indeed, several studies have shown that anxiety depression and somatization represent independent risk factors for the development of IBS postinfection ${ }^{30-32}$. Stress and other psychological factors may influence gut function contributing to symptom generation. A recent study showed the implications of stress in the expression of $\mathrm{T}$ helper 1 and T helper 2 (Th1/Th2) immune cells in PIIBS. The results of a study in 1379 subjects involved in a large outbreak waterborne infection caused by norovirus, G. lamblia, and Campylobacter, showed that anxiety, but not depression was associated with an increased risk of developing PIIBS. In addition, psychological factors involved in the development of PIIBS were in part due to an increased susceptibility to develop gastroenteritis. Those who had a Th2 type of response were more prone to develop PIIBS, suggesting an involvement of immune response in PIIBS pathogenesis $^{31}$. Figure 1 shows the main risk factor for the development of PIIBS. 


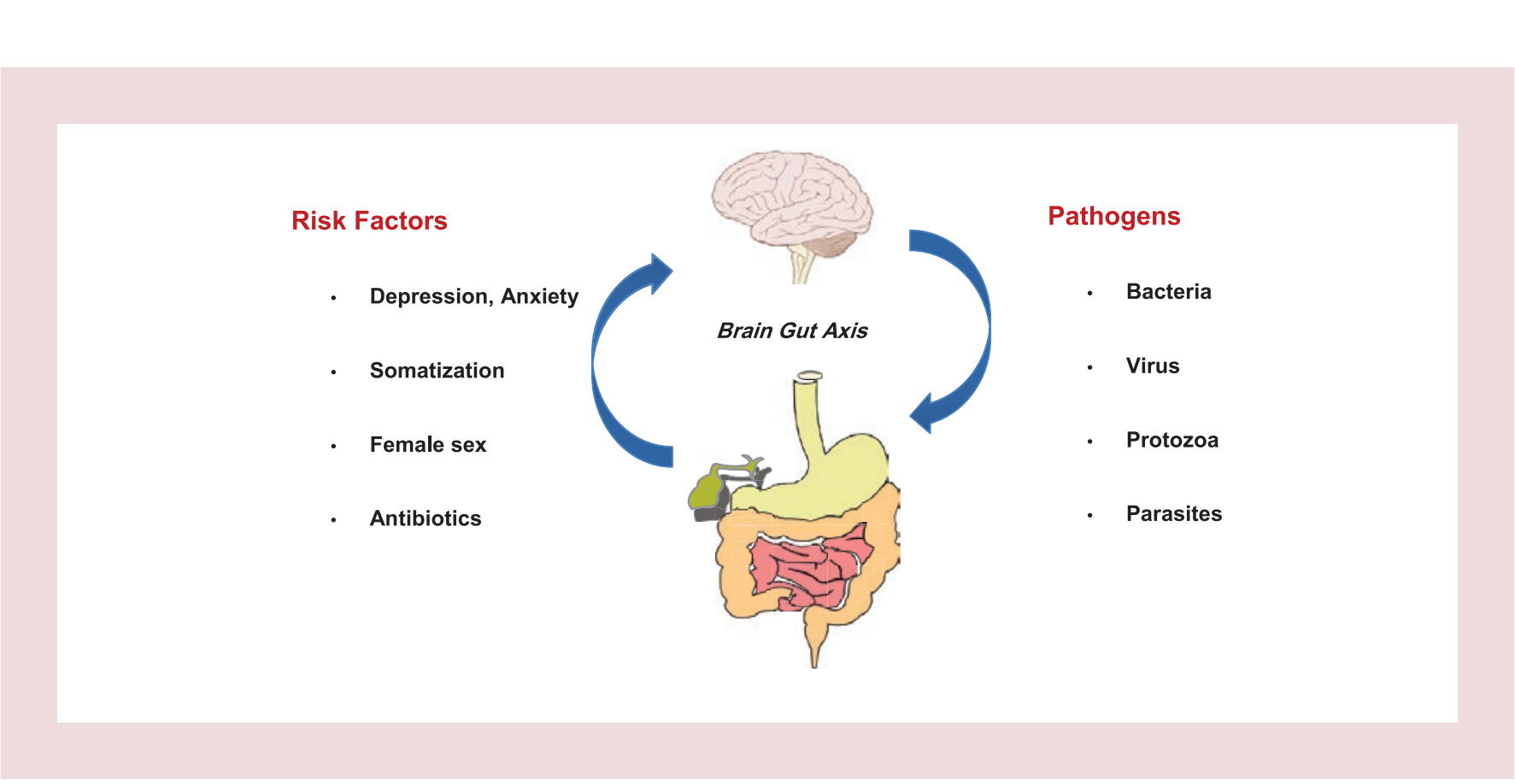

Figure 1. Main risk factors and etiologies of postinfection irritable bowel syndrome.

\section{MANAGEMENT}

Currently, there are limited data on specific management strategies and therapeutic approaches for PIIBS. A correct diagnosis of PIIBS should be made by fulfilling Rome IV criteria for IBS. In typical cases of PIIBS with no alarm features, a positive diagnosis without additional diagnostic tests is advisable. In limited severe cases or in those who do not respond to multiple therapeutic attempts, additional testing is encouraged. In some patients, particularly in those who have traveled in endemic regions for gastrointestinal parasites, it might be required to exclude chronic intestinal infestation such as Giardia infection with fecal stool cultures. Indeed, treatment of these infections would require specifically targeted therapies to eliminate the infectious agent. Exclusion of IBD simulating IBS could be investigated first with noninvasive methods such as the fecal calprotectin assay. This assay has shown a good sensitivity and high specificity to exclude IBD $^{33}$.

There are few studies testing the efficacy of pharmacological treatment in PIIBS. The role of prednisolone versus placebo was tested in a small group of patients with PIIBS. The results showed a reduction of intestinal enterochromaffin cell and lymphocyte counts in the rectal mucosa without any improvement in PIIBS symptoms ${ }^{34}$. In a further study conducted in a subset of 17IBS patients, the anti-inflammatory drug mesalazine have not shown improvement in the quality of life of patients and their reported symptoms ${ }^{35}$. A multicenter study testing the efficacy of mesalazine showed that this drug given during the acute phase of Shiga-like toxin producing E. coli O104:H4 infection had a protective effect on the development of PIIBS $^{36}$. Another double-blind randomized placebo-controlled trial confirmed the role of mesalazine in the improvement of abdominal 
pain severity and urgency in patients who developed IBS after acute infectious gastroenteritis $^{37}$. As reported in a recent review article $^{38}$, the immunological profile of patients would be of help to stratify the patients with IBS. This would, in turn, help to direct therapy.

Apart from data derived from these specific trials, the current therapeutic approach in PIIBS patients is adopted from currently available guidelines for IBS for which comprehensive reviews have been previously published in the literature ${ }^{1,39}$.

\section{CONCLUSION}

PIIBS represents a unique opportunity to study in a prospective manner the etiology and pathophysiology involved in the development of IBS. Bacteria, viruses, as well as protozoa and parasites are important triggers in PIIBS onset. The identification of populations at risk may help to identify strategies for the prevention of PIIBS. Long-term studies have shown that the prognosis of PIIBS could be more favorable than that of non-specific IBS; however, the identification of factors that induce the chronicity of symptom would be of importance. The diagnosis of PIIBS should not pose particular difficulties in typical patients developing IBS in the aftermath of an acute bout of infectious gastroenteritis of viral, bacterial, or parasitic infection. However, atypical cases represent a challenge for differential diagnosis and the management of severe symptoms. Anti-vinculin and anti-cytolethal distending toxin B antibodies are present in higher titer in IBS with diarrhea or mixed type and have been suggested to be of use in facilitating diagnosis ${ }^{40}$. In the future, the characterization of the microbiota both as a diagnostic and therapeutic tool could be of help.

In addition, there are still too limited data on the efficacy of non-pharmacological (dietary, prebiotics, and probiotics) approaches as well as pharmacological treatments.

\section{REFERENCES}

1. Mearin F, Lacy BE, Chang L, et al. Bowel Disorders. Gastroenterology. 2016 Feb 18. pii: S0016-5085(16)00222-5.

2. Barbara G, Feinle-Bisset C, Ghoshal UC, et al. The Intestinal Microenvironment and Functional Gastrointestinal Disorders. Gastroenterology. 2016 Feb 18. pii: S0016-5085(16)00219-5.

3. Klem F, Wadhwa A, Prokop LJ, et al. Prevalence, risk factors, and outcomes of irritable bowel syndrome after infectious enteritis: a Systematic review and meta-analysis. Gastroenterology. 2017;152:1042-540,

4. Spiller R, Garsed K. Postinfectious irritable bowel syndrome. Gastroenterology. 2009;136:1979-88.

5. Marshall JK, Thabane M, Garg AX, et al. Incidence and epidemiology of irritable bowel syndrome after a large waterborne outbreak of bacterial dysentery. Gastroenterology. 2006;131:445-50.

6. Tsuchiya $\mathrm{K}$. The significance of infectious disease and microbiota in functional gastrointestinal disorders. J Gen Fam Med. 2017;18:27-31.

7. Sundin J, Rangel I, Fuentes S, et al. Altered faecal and mucosal microbial composition in post-infectious irritable bowel syndrome patients correlates with mucosal lymphocyte phenotypes and psychological distress. Aliment Pharmacol Ther. 2015;41:342-51.

8. Jalanka-Tuovinen J, Salojärvi J, Salonen A, et al. Faecal microbiota composition and host-microbe cross-talk following gastroenteritis and in postinfectious irritable bowel syndrome. Gut. 2014;63:1737-45.

9. Saps M, Pensabene L, Di Martino L, et al. Post-infectious functional gastrointestinal disorders in children. J Pediatr. 2008;152:812-6, 816.e1.

10. Cremon C, Stanghellini V, Pallotti F, et al. Salmonella gastroenteritis during childhood is a risk factor for irritable bowel syndrome in adulthood. Gastroenterology. 2014;147:69-77.

11. Schwille-Kiuntke J, Frick JS, Zanger P, et al. Post-infectious irritable bowel syndrome-a review of the literature. Z Gastroenterol. 2011;49:997-1003.

12. Woese CR, Kandler O, Wheelis ML, et al. Towards a natural system of organisms: proposal for the domains Archaea, bacteria, and eucarya. Proc Natl Acad Sci U S A. 1990;87:4576-9.

13. Koonin EV, Senkevich TG, Dolja VV, et al. The ancient virus world and evolution of cells. Biol Direct. 2006;1:29.

14. Walker DM, Oghumu S, Gupta G, et al. Mechanisms of cellular invasion by intracellular parasites. Cell Mol Life Sci. 2014;71:1245-63.

15. Anganova EV, Savchenkov MF, Stepanenko LA, et al. Microbiological monitoring of opportunistic enterobacteriaceae of the Lena river. Gig Sanit. 2016;95:1124-8

16. Pawłowska B, Sobieszcza冈ska BM. Intestinal epithelial barrier: the target for pathogenic Escherichia coli. Adv Clin Exp Med. 2017;26:1437-45.

17. Roulston A, Marcellus RC, Branton PE, et al. Viruses and apoptosis. Annu Rev Microbiol. 1999;53:577-628. 
18. Guarner F, Malagelada JR. Gut flora in health and disease. The Lancet. 2003 Feb 8;361:512-9.

19. Balamurugan R, Janardhan HP, George S, et al. Bacterial succession in the colon during childhood and adolescence: molecular studies in a Southern Indian village. Am J Clin Nutr. 2008;88:1643-7.

20. Isolauri E, Salminen S, Ouwehand AC. Microbial-gut interactions in health and disease. probiotics. Best Pract Res Clin Gastroenterol. 2004; 18:299-313.

21. Andre G, Buret SV, Feener T, et al. Campylobacter jejuni-or Giardia duodenalis-mediated disruptions of human intestinal microbiota biofilms: novel mechanisms producing post-infectious intestinal inflammatory disorders. Gastroenterology. 2013;144:S309.

22. Chang L, Toner BB, Fukudo S, et al. Gender, age, society, culture, and the patient's perspective in the functional gastrointestinal disorders. Gastroenterology. 2006;130:1435-46.

23. Rodiño-Janeiro BK, Martínez C, Fortea M, et al. Decreased TESK1-mediated cofilin 1 phosphorylation in the jejunum of IBS-D patients may explain increased female predisposition to epithelial dysfunction. Sci Rep. 2018;8:2255.

24. Foster JA, Rinaman L, Cryan JF. Stress and the gut-brain axis: regulation by the microbiome. Neurobiol Stress. 2017;7:124-36.

25. Gorbach SL. Perturbation of intestinal microflora. Vet Hum Toxicol. 1993;35 Suppl 1:15-23.

26. Hurley BW, Nguyen CC. The spectrum of pseudomembranous enterocolitis and antibiotic-associated diarrhea. Arch Intern Med. 2002;162:2177-84.

27. Pimentel M, Lembo A, Chey WD, et al. Rifaximin therapy for patients with irritable bowel syndrome without constipation. N Engl J Med. 2011;364:22-32.

28. Pimentel M, Chow EJ, Lin HC. Eradication of small intestinal bacterial overgrowth reduces symptoms of irritable bowel syndrome. Am J Gastroenterol. 2000;95:3503-6.

29. Ponziani FR, Scaldaferri F, Petito V, et al. The role of antibiotics in gut microbiota modulation: the eubiotic effects of rifaximin. Dig Dis. 2016;34:269-78.
30. Van Oudenhove L. Bio psychosocial aspects of functional gastrointestinal disorders: how central and environmental processes contribute to the devolopment and expression of functional gastrointestinal disorder Gastroenterology. 2016;150:1355-67.

31. Wouters MM, Van Wanrooy S, Nguyen A, et al. Psychological comorbidity increases the risk for post infectious IBS partly by enhanced susceptibility to develop infectious gastroenteritis. Gut. 2016;65:1279-88.

32. Dunlop SP, Jenkins D, Neal KR, Spiller RC. Relative importance of enterochromaffin cell hyperplasia, anxiety, and depression in post infectious IBS. Gastroenterology. 2003;125:1651-9.

33. van Rheenen PF, Van de Vijver E, Fidler V. Faecal calprotectin for screening of patients with suspected inflammatory bowel disease: diagnostic meta-analysis. BMJ. 2010;341:c3369.

34. Dunlop SP, Jenkins D, Neal KR, et al. Randomized, double-blind, placebo-controlled trial of prednisolone in post-infectious irritable bowel syndrome. Aliment Pharmacol Ther. 2003;18:77-84.

35. Tuteja AK, Fang JC, Al-Suqi M, Stoddard GJ, Hale DC. Double-blind placebo-controlled study of mesalamine in post-infective irritable bowel syndrome-a pilot study. Scand J Gastroenterol. 2012;47:1159-64.

36. Andresen V, Löwe B, Broicher W, et al. Post-infectious irritable bowel syndrome (PI-IBS) after infection with Shiga-like toxin-producing Escherichia coli (STEC) O104:H4: a cohort study with prospective follow-up. United European Gastroenterol J. 2016;4:121-31.

37. Lam C, Tan W, Leighton $M$, et al. A mechanistic multicentre, parallel group, randomised placebo-controlled trial of mesalazine for the treatment of IBS with diarrhoea (IBS-D). Gut. 2016;65:91-9.

38. Bashashati M. Immune activation in irritable bowel syndrome: from basic to clinic. Neuro Gastro Latam Rev. 2017;1:116-27.

39. Moayyedi P, Mearin F, Azpiroz F, et al. Irritable bowel syndrome diagnosis and management: a simplified algorithm for clinical practice. United European Gastroenterol J. 2017;5:773-88.

40. Rezaie A, Park SC, Morales W, et al. Assessment of anti-vinculin and anti-cytolethal distending toxin B antibodies in subtypes of irritable bowel syndrome. Dig Dis Sci. 2017;62:1480-5. 